\title{
MAGNETIC PROPERTIES OF DyTe
}

\section{S. POKRZYWNICKI}

Department of Mathematics and Physics, Technical University of Opole Luboszycka 7, 45-036 Opole, Poland

\author{
M. Duczmal, L. Pawlak
}

Institute of Inorganic Chemistry and Metallurgy of Rare Elements Technical University of Wroclaw

Wyb. Wyspiańskiego 27, 50-370 Wrocław, Poland

\author{
AND K.A. NENKov \\ International Laboratory of High Magnetic Fields and Low Temperatures \\ Gajowicka 95, 53-529 Wroclaw, Poland
}

\begin{abstract}
Dysprosium monotelluride is a metallic substance swith one valence electron per formula unit belonging to the conduction band. It crystallizes in the defected $\mathrm{NaCl}$-type structure $(a=0.6070 \mathrm{~nm})$ with cation deficiency in the metal sublattice. AC and DC susceptibilities exhibit a maximum at $15 \mathrm{~K}$ and a second maximum at higher temperatures which position is field dependent.
\end{abstract}

PACS numbers: 75.50.Ee, 75.30.Cr

\section{Introduction}

The magnetic properties of the lanthanide chalcogenides are much less explored than the monopnictides. The main reasons are probably difficulties in syntheses and the crystals growth. In particular, the properties of monotellurides are almost completely unknown.

The dysprosium tellurides: DyTe and $\mathrm{Dy}_{2} \mathrm{Te}_{3}$, crystallize in the structures related to the rock salt type. The first compound is metallic, while the second semiconducting. Dysprosium sesquitelluride behaves like a simple antiferromagnet with the Neel temperature equal to $4.1 \mathrm{~K}[1]$. We synthesized and investigated the dysprosium monotelluride in order to examine a change of exchange interactions after transition to the metallic state. 


\section{Experimental}

Polycrystalline samples of dysprosium monotelluride were obtained by the direct synthesis from dysprosium metal (99.9\%, Koch Light Lab.) and tellurium $(6 \mathrm{~N}, \mathrm{POCH})$ in sealed quartz ampoules. After a reaction the mixture of dysprosium and dysprosium tellurides was ground in an argon box, pressed in pellets and sealed in the double quartz ampoules containing $200 \mathrm{~mm} \mathrm{Hg}$ of argon in the outer tube to prevent oxygen diffusion through the walls. The samples were heated at $1200 \mathrm{~K}$ by a week. This procedure was repeated twice.

Powder X-ray measurements were performed on the X'Pert Philips PW 1830 diffractometer using $\mathrm{Cu} K_{\alpha}$ radiation. The calculated lattice constant $a=$ $0.6070 \mathrm{~nm}$ is in good agreement with the literature data [2].

The magnetic susceptibility was measured in the temperature range $4.2-100 \mathrm{~K}$ in $133 \mathrm{~Hz} \mathrm{AC}$ field with static magnetic field up to $5 \mathrm{~T}$.

\section{Results and discussion}

The AC susceptibility plots measured in different static fields are shown in Fig. 1. Their characteristic feature is a presence of two maxima. The first of them appears about $15 \mathrm{~K}$ (it is denoted here as $T_{\mathrm{N}}$ ) and corresponds to an antiferromagnetic phase transition. It depends weakly on the DC magnetic field. The second maximum appears at higher temperatures and is strongly field dependent. Its position (46.5 $\mathrm{K}$ in zero magnetic field) shifts to lower temperatures when the static field increases and reaches the greatest height in $2 \mathrm{~T}$. Above $50 \mathrm{~K}$ the magnetic susceptibility follows the Curie-Weiss law with the Weiss constant $\Theta_{\mathrm{p}}=-10.1 \pm 0.5 \mathrm{~K}$. The effective paramagnetic moment at $100 \mathrm{~K}, \mu_{\mathrm{eff}}=9.4 \mu_{\mathrm{B}}$, is lower than the free ion value $\left(10.64 \mu_{\mathrm{B}}\right)$ for $\mathrm{Dy}^{3+}$. There are at least three possible reasons of the paramagnetic moment lowering: the crystal field (CF) influence, the screening effect of the conduction electrons and a nonstoichiometry. The CF only

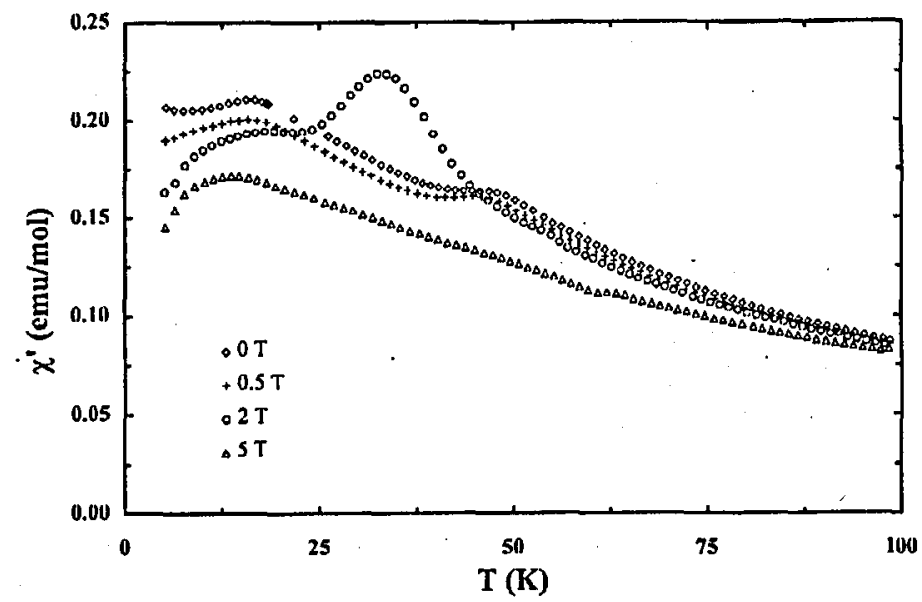

Fig. 1. The representative plots of $A C$ magnetic susceptibility of DyTe vs. temperature. 


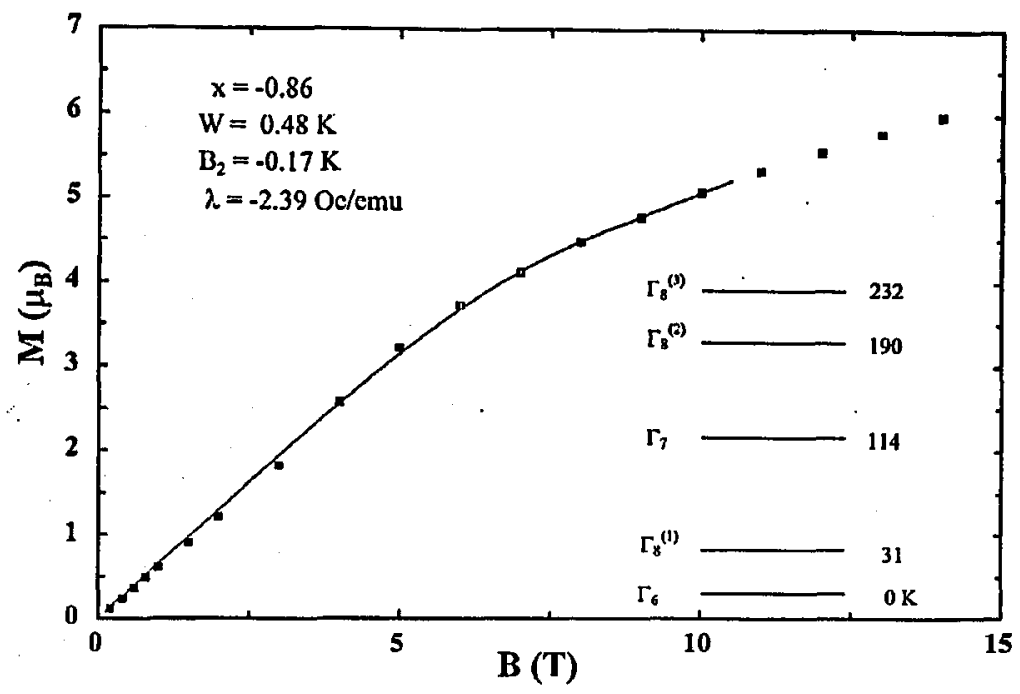

Fig. 2. Magnetization of DyTe vs. magnetic field at $4.2 \mathrm{~K}$. The solid line was calculated using the parameters given in the figure.

paramagnetic moment calculated at $100 \mathrm{~K}$ is close to the theoretical value. At this stage of the investigation we are not able to evaluate quantitatively the effect of screening. On the other hand, the cation deficiency in the metal sublattice (without changing the crystal structure) is common among the rare-earth monotellurides, e.g. for $\mathrm{Tb}_{x} \mathrm{Te}, x=0.85-0.95$, and for $\mathrm{IIo}_{x} \mathrm{Te}, x=0.91$ [3].

The magnetization was measured at $4.2 \mathrm{~K}$ in fields up to $14 \mathrm{~T}$ (Fig. 2). The experimental data were used to calculate the potential and energy levels of the ground multiplet $J=15 / 2$ in the crystal field. The isotropic exchange interactions (as a molecular field parameter $\lambda$ ) were taken into account. The details of the procedure were presented elsewhere [4]. $A$ better agreement between the calculated and the experimental magnetization was achieved after adding the second order axial term to the octahedral CF $H_{[001]}$ IIamiltonian. The origin of this term is not clear - that may be a small tetragonal distortion related to the deviation from the DyTe stoichiometry or quadrupolar exchange interactions. The following results were obtained (Lea, Leask and Wolf notation, dysprosium content $x=$ $0.87): W=0.46 \mathrm{~K}, x=-0.85, B_{2}=-0.17 \mathrm{~K}$ and $\lambda=-2.39 \mathrm{Oe} / \mathrm{emu}$.

The CF levels are presented in Fig. 2. The second order CF term splits each of the $\Gamma_{8}$ quartet into two doublets separated by $4-7 \mathrm{~K}$ (not shown in the figure). The overall ground term splitting $(232 \mathrm{~K})$ is lower than that obtained for the semiconducting $\mathrm{Dy}_{2} \mathrm{Te}_{3}(410 \mathrm{~K})[1]$, in spite of the larger Dy-Te distance $(0.3054 \mathrm{~mm})$ in the latter compound than in the former $(0.3035 \mathrm{~nm})$. The lowering of the CF splitting energy may be ascribed to a screening effect of conduction electrons.

The exchange interactions in DyTe are of antiferromagnetic type. The value of the molecular field parameter $\lambda(-2.39 \mathrm{Oe} / \mathrm{emu})$ is much larger than in $\mathrm{Dy}_{2} \mathrm{Te}_{3}$ 
$(\lambda=-0.112 \mathrm{Oe} / \mathrm{emu})$. As for $\mathrm{Dy}_{2} \mathrm{Te}_{3} T_{\mathrm{N}}=4.1 \mathrm{~K}$, then taking into account the dysprosium contents in both compounds, the Neel temperature of DyTe should be equal to $63 \mathrm{~K}$. The experimental value equal to $15 \mathrm{~K}$ is much lower than the expected one. The main reason of that difference is the cation deficiency, which increases the mean metal-metal distance and weakens the exchange interactions.

The origin of the second susceptibility maximum, occurring at higher temperatures $(30-50 \mathrm{~K})$ and strongly field-dependent, is unclear. An explanation of its nature needs further investigations.

\section{References}

[1] S. Pokrzywnicki, J. Alloys Comp. 225, 163 (1995).

[2] N.Ch. Abrikosov, K.A. Zinchenko, A.A. Eliseev, Izv. Akad. Nauk SSSR, Neorg. Mater. 6, 720 (1970).

[3] P. Fischer, P. Schobinger-Papamantellos, E. Kaldis, A. Erust, J. Phys. C, Solid Stale Phys. 10, 3601 (1977) and 11, 2653 (1978).

[4] M. Duczmal, S. Pokrzywnicki, L. Pawlak, Acta Phys. Pol. A 77, 687 (1990). 